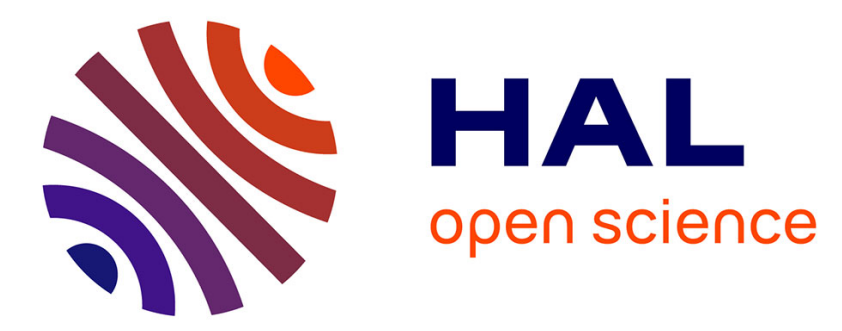

\title{
Plasmatic Signature of Disease by Differential Scanning Calorimetry (DSC)
}

Philipp A Tsvetkov, François Devred

\section{To cite this version:}

Philipp A Tsvetkov, François Devred. Plasmatic Signature of Disease by Differential Scanning Calorimetry (DSC). Microcalorimetry of Biological Molecules: Methods and Protocols, Springer, pp.45-57, 2019, 978-1-4939-9179-2. 10.1007/978-1-4939-9179-2_4. hal-02088538

\section{HAL Id: hal-02088538 \\ https://hal-amu.archives-ouvertes.fr/hal-02088538}

Submitted on 3 Apr 2019

HAL is a multi-disciplinary open access archive for the deposit and dissemination of scientific research documents, whether they are published or not. The documents may come from teaching and research institutions in France or abroad, or from public or private research centers.
L'archive ouverte pluridisciplinaire HAL, est destinée au dépôt et à la diffusion de documents scientifiques de niveau recherche, publiés ou non, émanant des établissements d'enseignement et de recherche français ou étrangers, des laboratoires publics ou privés. 


\title{
Plasmatic Signature of Disease by Differential Scanning Calorimetry (DSC)
}

\author{
Philipp 0. Tsvetkov and François Devred
}

\begin{abstract}
Differential scanning calorimetry (DSC) has been used for several decades to characterize thermal stability of macromolecules such as proteins and DNA. It allows to determine the denaturation temperature and enthalpy of individual domains of proteins, thus giving new insights into their domain organization and ligand interaction. Over the past decade, it has been shown that this technique can also be used to study biofluids such as plasma or cerebrospinal fluid to obtain denaturation profiles. An increasing number of studies demonstrated that such profiles obtained from patients were significantly different from profiles obtained using biofluids of healthy individuals. This opens interesting perspectives for new diagnostics and monitoring tools for a large number of diseases. Nevertheless, the extensive studies of plasma samples from patients with different pathologies as well as the development of standardized methods of data analysis are necessary to reach the promising diagnostic potential of this methodology. Using plasma samples from healthy individuals and glioblastoma patients, we outline the steps necessary to obtain a plasmatic calorimetric profile with VP-DSC instrument and describe a cluster analysis of obtained data.
\end{abstract}

Key words Differential scanning calorimetry, Plasma, Disease, Glioblastoma

1 Introduction

Differential scanning calorimetry (DSC) is a powerful biophysical method which in its conventional use characterizes the stability of a purified protein or other biomolecule directly in solution. It measures the heat exchange associated with the molecule's thermal denaturation when heated at a constant rate $[1,2]$. It has several important applications including quality control of pharmaceutical preparations, nanoparticle stability, tertiary structure characterization, and interaction studies [3]. For purified single-domain proteins, the profile of denaturation obtained by DSC is usually characterized by a single peak. The maximum of this peak corresponds to the melting temperature $(\mathrm{Tm})$ and the area under the curve to the enthalpy of denaturation $(\Delta H)$. These two parameters are characteristic for each protein at given conditions. Any 
modification in the protein structure such as mutations, posttranslational modifications, or interaction with any kind of ligand could impact protein stability and thus be detected using DSC.

More recently, a less conventional use of DSC has been proposed. It consists in applying DSC directly to biofluids, such as serum, plasma, or cerebrospinal fluid, rather than to purified biomolecules. The denaturation profiles of biofluids obtained by DSC correspond to the superposition of denaturation calorimetric profiles of all components in the biofluid sample.

The modifications in blood composition of patients suffering from a disease can lead to significant and reproducible changes in calorimetric profile of plasma corresponding to specific signature of the disease. Just like for purified proteins, the change in profile can be due to any of the modifications mentioned above (mutation, ligand, posttranslational modification) or the change in concentration of circulating proteins.

Over the past 5 years, an increasing number of publications have shown that such impact exists in many diseases including several types of cancer [4-10].

In this chapter we describe the straightforward way to obtain plasmatic thermograms by DSC taking as an illustration the profiles of healthy individuals and patients suffering from glioblastoma. We also present how these profiles can be analyzed using cluster analysis.

\section{Materials}

\subsection{Plasma Sample Preparation}

\subsection{Sample Preparation}

\subsection{Obtention of Plasma DSC Profiles}

Prepare all solutions using ultrapure water and analytical grade reagents. All consumables that are in contact with plasma should be recycled properly, and all instruments that are in contact with plasma should be sterilized.

1. Lavender cap vacutainers are used to collect blood and prepare plasma. They are commercially treated with EDTA to prevent coagulation of the blood ( see Note $\mathbf{1}$ ).

2. Heraeus Multifuge X3R centrifuge equipped with TX 1000 swinging buckets.

1. To prepare $100 \mathrm{~mL}$ of dilution buffer, add $0.33 \mathrm{~g}$ of trisodium citrate to final volume of $100 \mathrm{~mL}$ of phosphate-buffered saline (PBS).

2. Polypropylene cryovials and Eppendorf tubes.

1. DSC thermograms are collected using a differential scanning VP-DSC microcalorimeter (MicroCal, Malvern Panalytical) or any analog instrument. 
2. Associated instruments such as ThermoVac (MicroCal) and cell cleaning apparatus are used to facilitate degassing samples and cleaning of the cells.

3. Origin 7 software (OriginLab Corporation, Northampton, MA) is used to treat raw data and obtain thermograms.

4. Hamilton syringe to fill the calorimetric cells.

2.4 Cluster Analysis

1. Wolfram Mathematica 11.0 software.

2. Microsoft Excel software.

3 Methods

3.1 Plasma

Preparation

3.2 Sample

Preparation
Carry out all procedures at room temperature unless otherwise specified.

Plasma is produced when whole blood is centrifuged in tubes that are treated with an anticoagulant. The blood does not clot in the plasma tube. The cells are removed by centrifugation. All plasma sample manipulations should be carried out with appropriate precautions using gloves and throwing all consumables in dedicated yellow trash boxes.

1. Collect whole blood into EDTA-treated vacutainer (lavender cap).

2. Equilibrate the tubes in the TX 1000 rotor of the $\mathrm{X} 3 \mathrm{R}$ centrifuge.

3. Centrifuge $1000 \times g$ at $20-25^{\circ} \mathrm{C}$ for $20 \mathrm{~min}($ see Note 2$)$.

4. Following centrifugation, it is important to immediately transfer the liquid component (plasma) into a clean polypropylene tube by aspiration ( see Note $\mathbf{3}$ ).

5. The samples should be maintained at $2-8{ }^{\circ} \mathrm{C}$ while handling. If the plasma is not analyzed immediately, the plasma should be separated into $0.5 \mathrm{~mL}$ aliquots and stored at $-20^{\circ} \mathrm{C}$ or lower.

6. Unprocessed plasma samples are stored at $-80^{\circ} \mathrm{C}$ ( see Note 4$)$.

1. Before DSC analysis, thaw the samples rapidly at $37^{\circ} \mathrm{C}$, and then pipet $40 \mu \mathrm{L}$ of plasma into a final volume of $1 \mathrm{~mL}$ of dilution buffer.

2. Diluted samples can be stored at $-20{ }^{\circ} \mathrm{C}$ when not in use. 


\subsection{Obtention of Plasma DSC Profiles}

3.3.1 Instrument Settings

3.3.2 Cell Filling

and Running Experiment
Configure DSC instrument using control software and the following settings:

1. Number or scans: 2 .

2. Post-cycle thermostat: 10 .

3. Cell concentration $(\mathrm{mM})$ : 1 ( see Note 5).

4. Starting temperature $\left({ }^{\circ} \mathrm{C}\right): 10$.

5. Final temperature $\left({ }^{\circ} \mathrm{C}\right): 120$.

6. Scan rate $(\% /$ h. $): 60$.

7. Prescan thermostat (min): 15 .

8. Postscan thermostat (min): 0 .

9. Filtering period: 2 .

10. Feedback mode/gain: Mid.

All manipulations with calorimetric cell should be performed with extra care to avoid damaging cell with filling syringe and contamination with biofluids (always use gloves when working with plasma samples).

1. Fill the Hamilton syringe with at least $580 \mu \mathrm{L}$ of buffer at room temperature (see Note 6).

2. To degas the sample, block the tip of the syringe, and slowly pull the piston up to twice the volume of the sample. Release slowly the piston to initial position. Repeat three times.

3. Insert the filling funnel into expansion on the top of the calorimetric cell.

4. Introduce the filling syringe into calorimetric cell through filling funnel.

5. To inject in the calorimetric cell, push the syringe piston slowly until half of solution volume is injected, and then inject the rest by doing several small but rapid injections. This should be done while moving the syringe toward the top of the cell (see Note 7).

6. Proceed with the injection up to the point when the sample overflowing the calorimetric cell ( see Note 8).

7. Remove this overflowing liquid with the syringe.

8. Close the instrument by rotating first the metal and then plastic part of the cap ( see Note 9).

9. Select "DSC instruments" in the main window tab selections.

10. Verify instrument settings (see above).

11. Press start in the main window buttons (see Note 10). 
Between each plasma sample analyzed, the cell must be cleaned extensively to prevent attachment of aggregates on the cell walls (see Note 11). For this purpose, the ThermoVac in conjunction with its cell cleaning device can be used to circulate cleaning and rinsing solutions through the calorimetric cells.

1. Clean the cell as follows:

(a) Empty the cell with a dedicated syringe.

(b) Fill it with Decon 10-20\% by syringe; push-pull piston few times.

(c) Remove Decon.

(d) Wash cell with 50-100 mL water using the ThermoVac (see Note 12).

(e) Rinse three times with buffer (see Note 13).

2. Extra cleaning:

(a) Fill the cell with Decon detergent 20\% (see Note 14).

(b) Select "Thermostat/Calib." in the main window tab selections to set the jacket temperature at $60{ }^{\circ} \mathrm{C}$.

(c) Leave for $1 \mathrm{~h}$ and then change the jacket temperature to $25{ }^{\circ} \mathrm{C}$.

(d) Leave overnight.

(e) Next morning empty the calorimetric cell and use the normal cleaning procedure.

(f) If this procedure is still not enough, Decon $20 \%$ can be replaced with $5 \% \mathrm{NaOH}$ ( see Note 15 ).

3. Clean filling funnel by rinsing it with excess of water and methanol, and then sterilize it.

4. Clean filling syringe by rinsing it with excess of water and methanol, and then sterilize it.

3.4 Raw Data Processing
1. Thermograms are treated using software Origin 7.

2. Select "read data," select the two files corresponding to the two scans, and click "Add files" and then "Ok" (Fig. la).

3. In the top menu, select "Math" and then "simple math."

4. In the pop-up window, select the first scan name in the field "Yl" and the second scan name in the field "Y2" by scrolling "Available data" list and pressing corresponding button with arrow " $\Rightarrow$ ". Then put “-” (minus) sign in "Operator" field and press "Ok" (Fig. 1b, black solid curve).

5. At this point, the resulting scan replaced the first one so you can remove the second scan.

6. Peak $>$ Start Baseline Session; red segments will appear on both sides of the thermogram (Fig. 1b, red segments). 

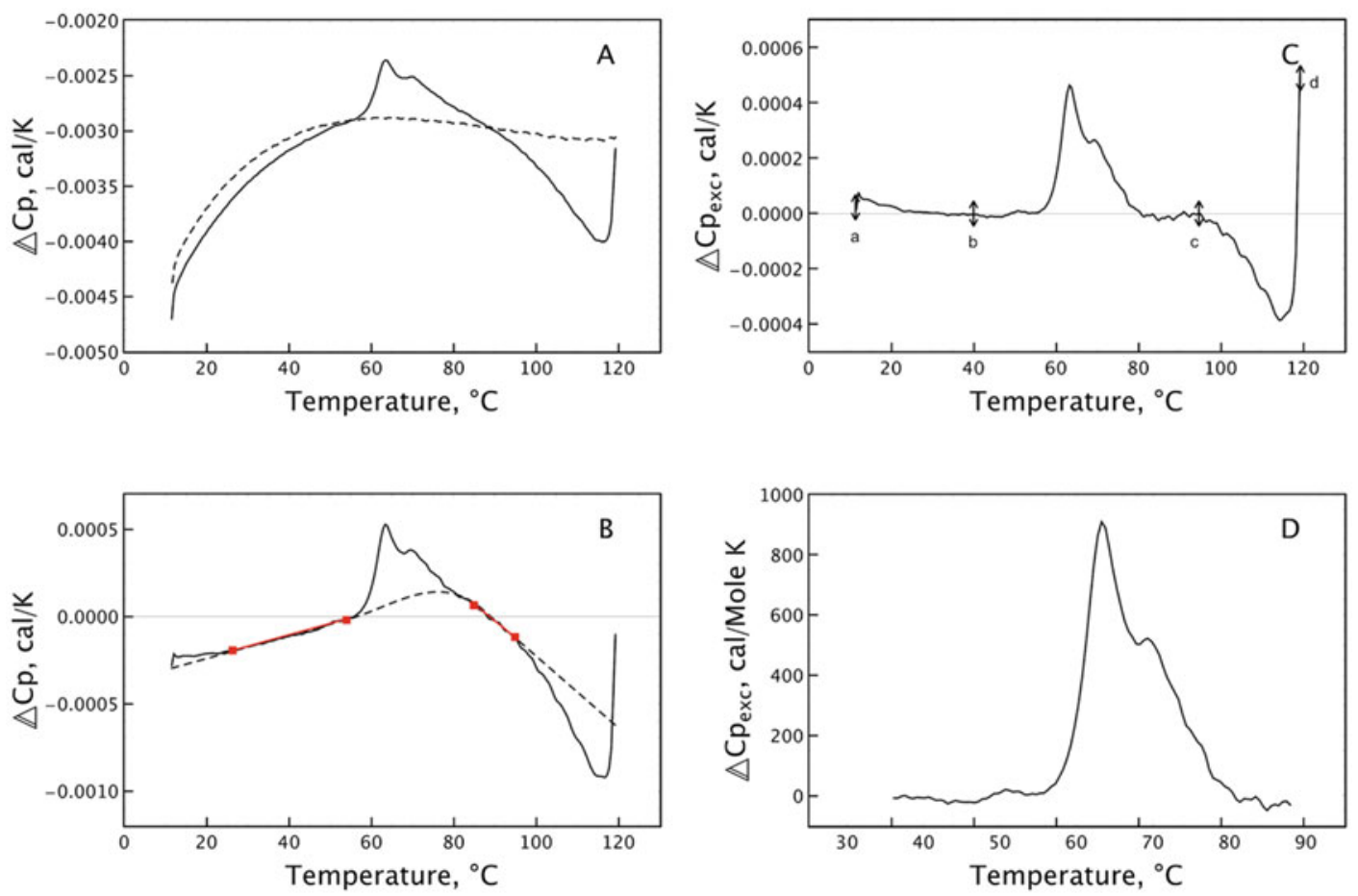

Fig. 1 Stages of raw DSC data processing. (a) First (solid curve) and second (dashed curve) DSC scans. (b) Difference between first and second scan (solid black curve), segments for baseline creation (red lines and squares), and created baseline (dashed curve). (c) Experimental curve with subtracted baseline and fragments of the curve marked with double-arrowed cursors a-b and c-d that should be removed. (d) Final excess heat capacity curve after concentration normalization

7. Adjust $>$ move segments by cursors; match the red segments with the segment of the experimental curve you want to use for baseline usually $10-40{ }^{\circ} \mathrm{C}$ and $84-100{ }^{\circ} \mathrm{C}$ (see Note 16) and press "enter."

8. Select "Baseline" with "cubic connect" and press OK (Fig. Ib, black dashed curve).

9. Confirm that you want to subtract baseline (Fig. 1c, black solid curve).

10. From the Tool menu under the graphic, use the "data selector" to choose the segment of the curve that will not be analyzed (0-40 ${ }^{\circ} \mathrm{C}$ and $\left.100-120{ }^{\circ} \mathrm{C}\right)$, and press “enter" to validate selection and "suppr" to delete it (Fig. lc).

11. In the side menu, select "Normalize concentration," put " 1 ," and click "Ok" (Fig. 1d) (see Note 17).

12. In the top menu, click "Window" to select the processed curve; that will open you the table of values.

13. Select File Export To Ascii and save your data as a .DAT file. 

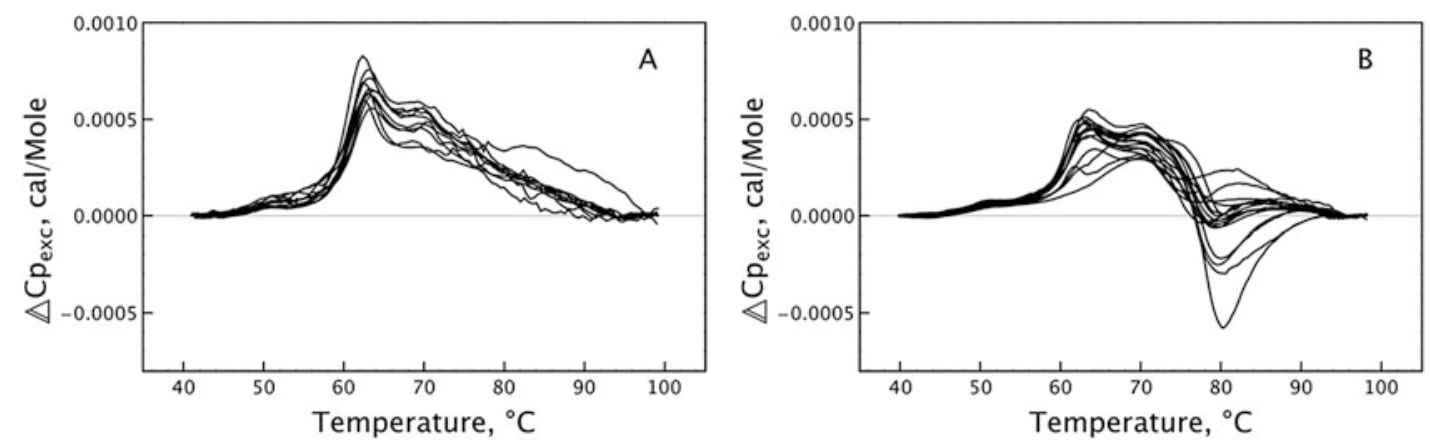

Fig. 2 Calorimetric profiles of plasma obtained from 10 healthy individuals (a) and 17 GB patients (b)

3.5 Cluster Analysis The general information about cluster analysis can be found in the following publications $[11,12]$.

1. Average thermograms obtained from the patients and healthy individuals and patients, and find the difference between them. For that purpose:

(a) Create folder and copy to it all DSC “* .dat” files obtained from healthy individuals (Fig. 2a).

(b) Create folder and copy to it all DSC “* .dat” files obtained from patients (Fig. 2b).

(c) Create new Notebook in Mathematica software; copy in it the code from Annex 1.

(d) Replace \#\#Dirl\#\# and \#\#Dir2\#\# in the code (lines 21 and 26) by paths to newly created folders (see Note 18), and replace \#\#FigureDir\#\# (line 71) by path to folder where you want to save the figure file.

(e) Modify if necessary the parameters in the code (see Note 19).

(f) Press "Shift+Enter" to run the code (see Note 20). In directory \#\#FigureDir\#\# new ready-to-publish image file "FigureDSC.png" with resulting averaged curves and their difference will be created (Fig. 3).

2. From difference curve drawn at the bottom panel of obtained figure, determine at what temperature the thermograms of patients are most different from the ones of healthy individuals by defining the positions of the extremums of difference curve (see Note 21).

3. Create Excel file and fill its table with the values of each experimental curve at temperatures chosen at previous step.

4. Add to table the columns with other characteristics of the experimental curves, such as positions and values of the maxima of each curve, surface under the curves, or any other 


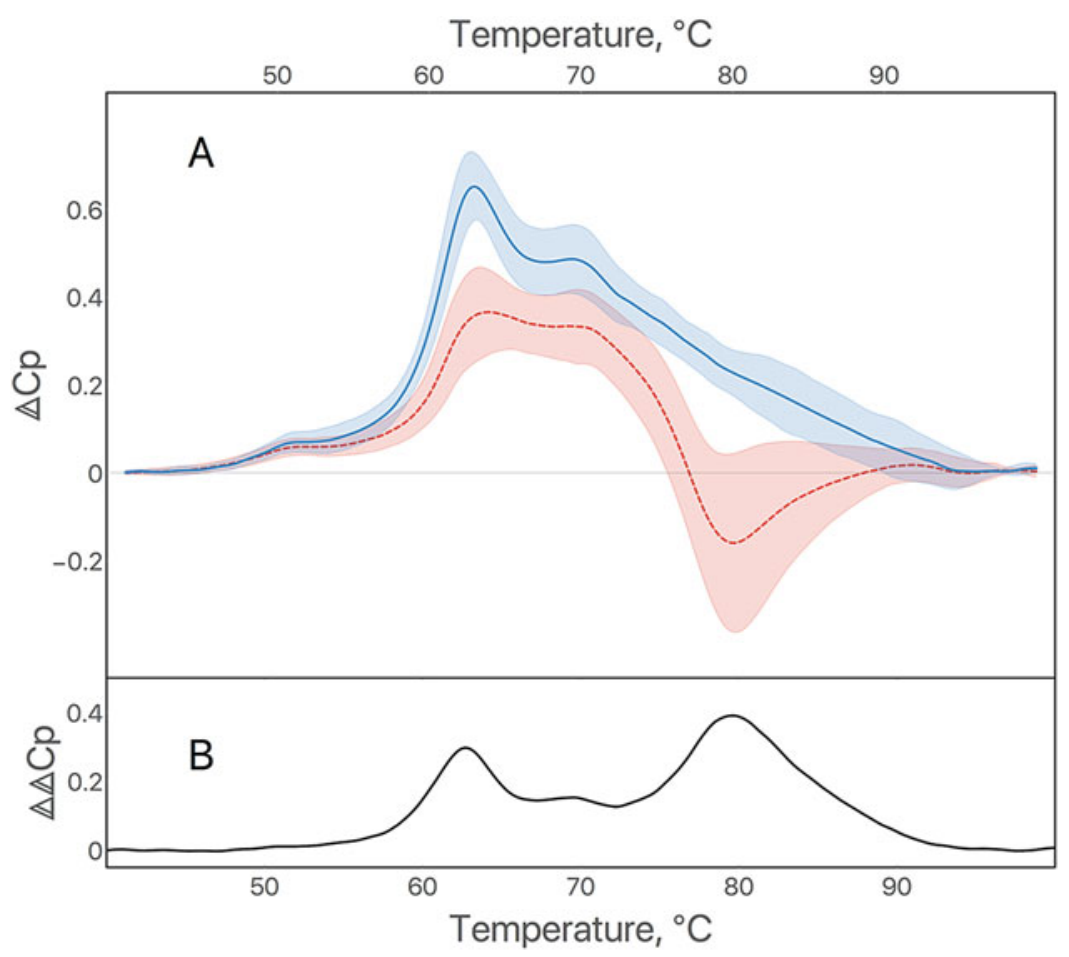

Fig. 3 The example image generated by code from Annex 1 in Wolfram Mathematica software. In this example (a) represents the average of plasma denaturation profiles from 10 healthy individuals (blue solid curve) and 17 GB patients (red dashed curve). Filled area corresponds to standard deviation. (b) Difference between averages of plasma denaturation profiles from healthy individuals and GB patients

parameters of the curves you believe could differ profiles of healthy individuals and patients.

5. Save resulting table in "DSC_Data.xlsx" file.

6. Perform cluster analysis of these data for that purpose:

(a) Create new Notebook in Mathematica software; copy in it the code from Annex 2.

(b) Replace in the code \#\#Dir_of_xls_file\#\# at line 1 by the path to directory where you saved "DSC_Data.xlsx" file.

(c) Replace in the code \#\#FigureDir\#\# at line 31 by the path to directory where you want to save the resulting figure file with clusters ( see Note 18).

(d) Modify if necessary the number of clusters set by default equal to 4 in line 5 (parameter numberOfClusters) and clustering method in line 8 (parameter Method $\rightarrow$ "Optimize"). For options of clustering, see online Wolfram Language documentation [13]. Modify if necessary any other parameters in the code (see Note 19). 


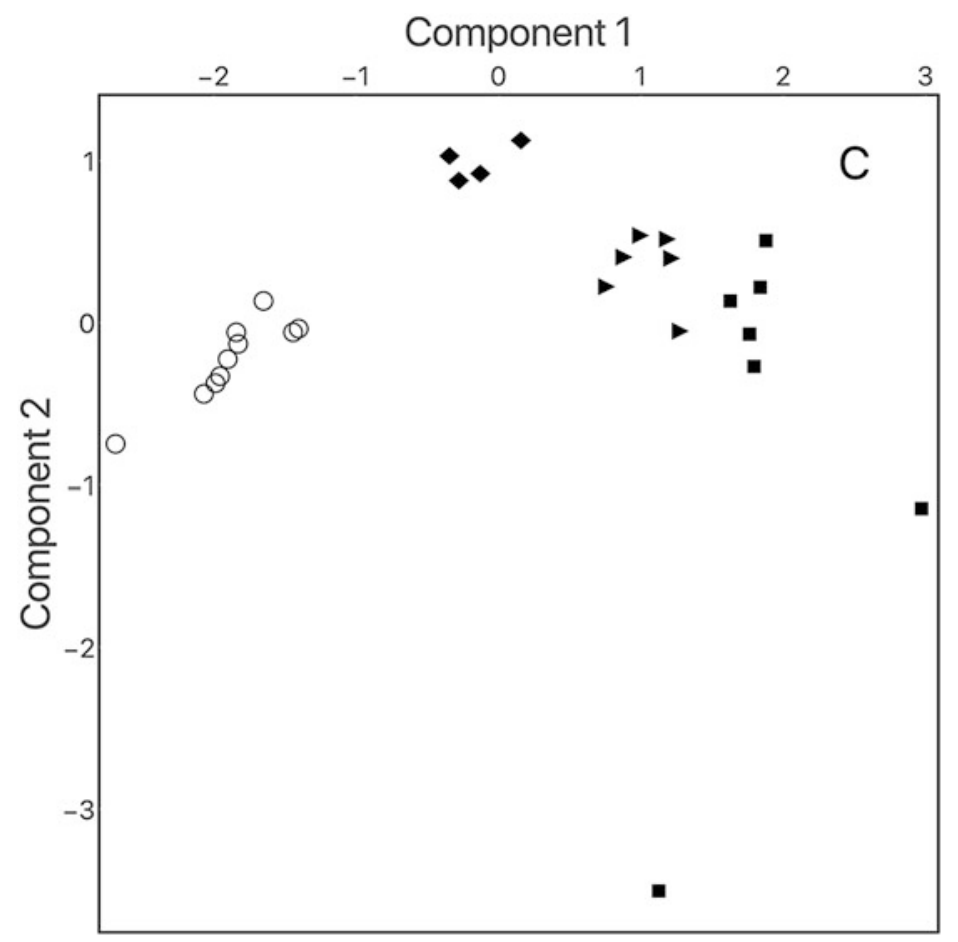

Fig. 4 The example image generated by code form Annex 2 by Wolfram Mathematica software. In this example, all curves were divided into four distinct clusters, wherein open circles correspond to healthy individuals and filled shapes to glioblastoma patients

(e) Press "Shift+Enter" to run the code (see Note 20). In directory \#\#FigureDir\#\# new Clusters.png file with results of cluster analysis will be created (Fig. 4).

\section{Notes}

1. Citrate-treated (light blue caps) could probably be used too, but heparinized tubes (green tops) can be contaminated with endotoxin, which can stimulate white blood cells to release cytokines.

2. Samples should not be put on ice; otherwise they will hemolyse.

3. If supernatant appears too pink, it is probably a sign of hemolysis which will invalidate the obtained profile.

4. No other specific purification step is added in order to avoid perturbing the interactome or alter the chemical state of plasma proteins. 
5. Value of 1 is put arbitrarily because one cannot perform an experiment if concentration is not specified.

6. Even though the cell volume is only $400 \mu \mathrm{L}, 580 \mu \mathrm{L}$ is necessary to guarantee the absence of bubbles in the cell.

7. Short rapid injections will help remove eventual bubbles attached to the cell walls.

8. This procedure also decreases the chances of having bubbles trapped in the cell.

9. Verify that the pressure increases upon rotation of the plastic cap and reaches 30 psi when the rotation is finished. This will prevent sample boiling at high temperatures.

10. After the first run is completed, the instrument will cool down the sample and launch the second run automatically.

11. If the cleaning is not done properly, the signal will become more and noisier after each experiment.

12. See manufacturer protocol for ThermoVac use.

13. At this point, you can run a buffer-buffer experiment; if the signal is noisy, you can then use the extra cleaning procedure.

14. When using detergents or corrosive solution, the pressure transducer must be closed with its dedicated white plug.

15. For other stronger cleaning procedure, see VP-DSC manufacturer manual.

16. Since all samples aggregate starting from $100{ }^{\circ} \mathrm{C}$, the segment on the right should stop before.

17. Global protein concentration is not normalized so that the profiles obtained reflect the possible differences in protein content of plasma.

18. You can do so by holding "Alt" key and dragging and dropping the directory in the desired place in the Notebook.

19. For quick guide to Mathematica code, see ref. [14].

20. The execution of the code could take several minutes.

21 . In our case, there are two extremums; it could be also more.

The plasma from patients belonging to the Marseille glioma cohort (CPP number: 2014-A00585-42) was retrieved from the AP-HM tumor bank (AC-2013-1786/CRB number BB-0033-00097). Thanks to the patient association ARTC Sud for supporting the project "Vers la mise au point d'un outil de suivi non invasif des gliomes." This work has been also supported by the Fondation ARC pour la recherche sur le cancer. 


\section{Annex 1}

1. drawCurves [data0_, colro_, style0_] :=

2. Module [

3. $\quad\{$ data $=$ data $0, \operatorname{colr}=\operatorname{colr} 0$, style $=$ style0 ,

4. filetredLists = Transpose / ( ( \#1, WienerFilter [\#2, 1, .1] $\}$ \& @@@ Transpose /@ data);

5. $\quad \mathrm{ip}=$ Interpolation / @ filetredLists;

6. average $\left[\mathrm{x}_{-}\right]:=$MeanaThrough [ip[x]];

7. $\operatorname{sd}\left[\mathrm{x}_{-}\right]:=$StandardDeviation@Through $[\mathrm{ip}[\mathrm{x}]]$;

8. maximal[x_] := Max@Through [ip[x]];

9. $\operatorname{plt} 1=$ Plot $[$ average $[\mathrm{x}],\{\mathrm{x}, 40,100\}$, Plotstyle $->$ \{Thick, colr, style $\}$;

10. pltsd $=\operatorname{Plot}[\mathrm{sd}[\mathrm{x}],\{\mathrm{x}, 40,100\}$, PlotStyle $->$ TThick, colr $\}]$;

11. $\operatorname{plt} 2=\operatorname{Plot}[\{\operatorname{average}[\mathrm{x}]-\operatorname{sd}[\mathrm{x}]$, average $[\mathrm{x}]\},\{\mathrm{x}, 40,100\}$,

12. Filling $->\{1->\{2\}\}$, Plotstyle $->\{\{$ Thin, colr $\},\{$ Thin, colr $\}$,

13. Fillingstyle $->\{$ colr, Opacity[0.2]\}];

14. $\operatorname{plt} 3=\operatorname{Plot}[\{\operatorname{average}[\mathrm{x}]$, average $[\mathrm{x}]+\operatorname{sd}[\mathrm{x}]\},\{\mathrm{x}, 40,100\}$, Filling $-\& g t ;\{1-$ \&gt; $\{2\}\}$,

15. Plotstyle $->\{\{$ Thin, colr $\},\{$ Thin, colr $\}$,

16. Fillingstyle $->$ \{colr, Opacity[0.2]\}];

17. Show [plt1, plt2, plt3, Framestyle-> Thickness[0.0015]]

18. ] ;

19 .

20.

21. files1 = FileNames ["*.DAT", "\#\#Dir1\#\#"];

22. data1 = Drop [Import [\#], 40] \&/@ files1;

23. $\operatorname{cv1}=$ drawCurves [data1, RGBColor $[0.91,0.34,0.247]$, Dashed];

24 .

25.

26. files2 = FileNames ["*.DAT", "\#\#Dir2\#\#"];

27. data2 = Drop[Import $[\#], 40] \& /$ a files 2 ;

28. $\operatorname{cv} 2=$ drawCurves $[$ data2, $\operatorname{RGBColor}[0.29,0.573,0.86]$, Solid];

29.

30 .

31. getDataAv[data0_] := Module [

32. $\{$ data $=$ data 0$\}$,

33. filetredLists $=$ Transpose / @ ( \{\#1, WienerFilter $[\# 2,1, .1]\}$ \& @@@ Transpose / @ data);

34. $i p=$ Interpolation / a filetredLists;

35. average $\left[\mathrm{x}_{-}\right]:=$Mean@Through $[\mathrm{ip}[\mathrm{x}]]$;

36. Return [average $[\mathrm{x}]]$

37. ]

38 .

39. aa $\left[x_{-}\right]:=\operatorname{getDataAv}[$ data2 $]$ - getDataAv[data1];

40. difPlot $=$

41. Plot $[a a[x],\{x, 40,100\}, P l o t S t y l e->\{$ Thick, Black\},

42. PlotRange $->\{\{40,100\},\{-0.00005,0.0005\}\}$,

43. Framestyle $->$ Thickness [0.0015]]; 
44.

45. dscCurves $=$

46. Column [

47. $\{$ Show [

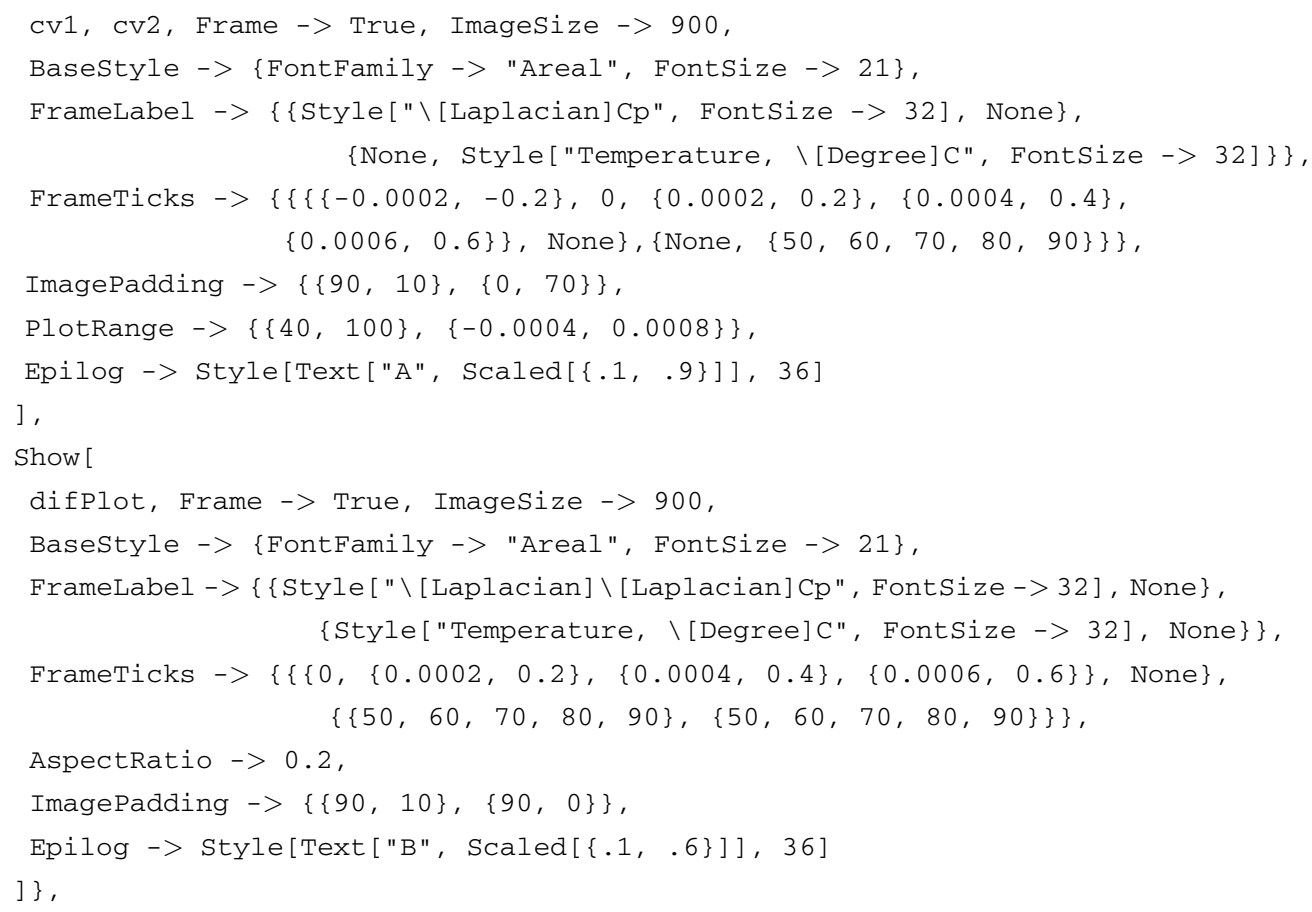

71. Export ["\#\#FigureDir\#\#/FigureDSC.png", \%, ImageResolution -> 300]

\section{Annex 2}

1. data $=$ Import ["\#\#ir_of_xls_file\#\#/DSC_Data.xlsx"] [ [1] ];

2. fdata $=$ data // Standardize;

3. paramsN $=$ Length $[$ fdata [[1] ] -5 ;

4.

5. numberOfClusters $=4$;

6.

7. builtinscores = PrincipalComponents [fdata, Method $->$ "Covariance"];

8. Clasters $=$ FindClusters[builtinscores, numberOfClusters, Method -> "Optimize"];

9 .

10. clasters $=$

11. Show [

12. clustersPlot $=$ ListPlot [

13. Clasters[[All, All, 1 ; ; 2]], PlotRange -> All, ImageSize -> 730,

14 . 


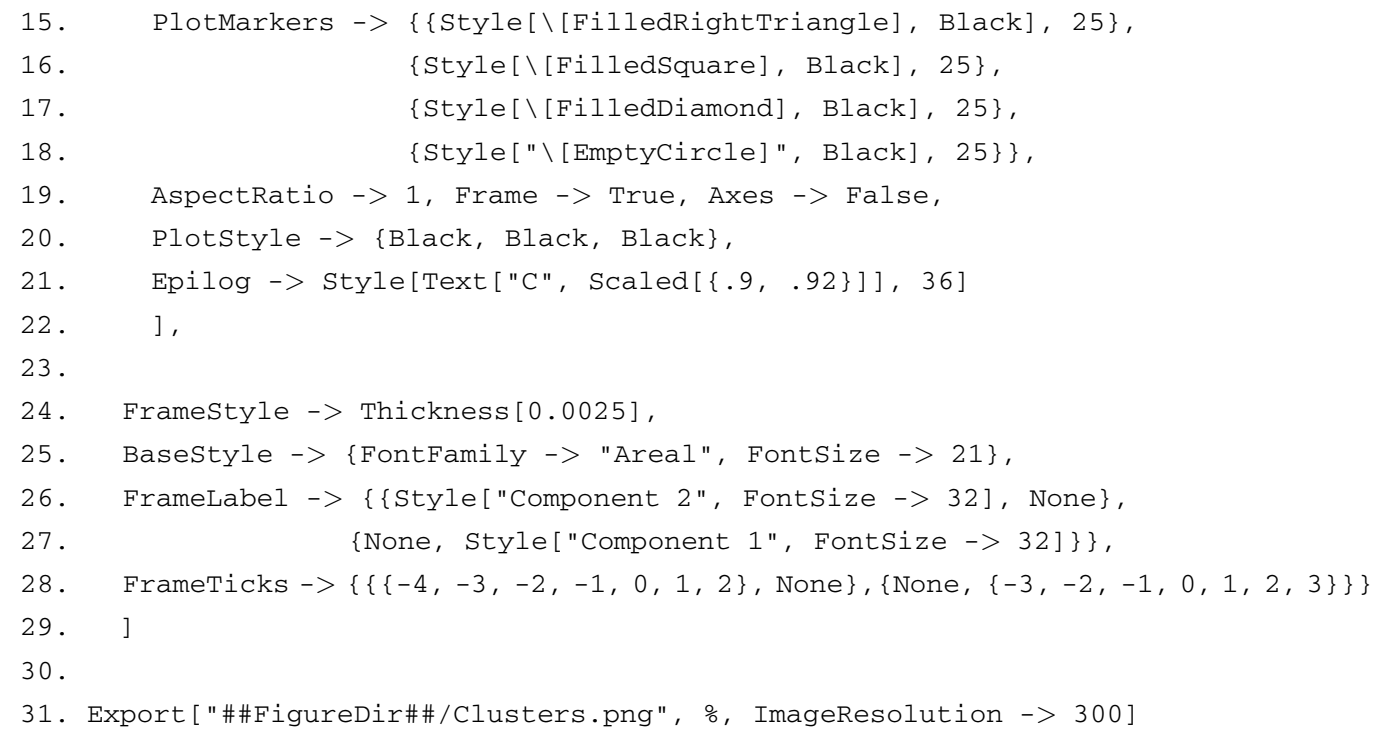

\section{References}

1. Petrushanko IY, Lobachev VM, Kononikhin AS, Makarov AA, Devred F, Kovacic H, Kubatiev AA, Tsvetkov PO (2016) Oxidation of $\mathrm{Ca} 2+$-binding domain of NADPH oxidase 5 (NOX5): toward understanding the mechanism of inactivation of NOX5 by ROS. PLoS One 11:e0158726

2. Roman AY, Devred F, Lobatchov VM, Makarov AA, Peyrot V, Kubatiev AA, Tsvetkov PO (2016) Sequential binding of calcium ions to the B-repeat domain of SdrD from Staphylococcus aureus. Can J Microbiol 62:123-129

3. Tsvetkov PO, Ezraty B, Mitchell JK, Devred F, Peyrot V, Derrick PJ, Barras F, Makarov AA, Lafitte D (2005) Calorimetry and mass spectrometry study of oxidized calmodulin interaction with target and differential repair by methionine sulfoxide reductases. Biochimie $87: 473-480$

4. Garbett NC, Mekmaysy CS, DeLeeuw L, Chaires JB (2015) Clinical application of plasma thermograms. Utility, practical approaches and considerations. Methods 76:41-50

5. Garbett NC, Miller JJ, Jenson AB, Miller DM, Chaires JB (2007) Interrogation of the plasma proteome with differential scanning calorimetry. Clin Chem 53:2012-2014

6. Garbett NC, Mekmaysy CS, Helm CW, Jenson AB, Chaires JB (2009) Differential scanning calorimetry of blood plasma for clinical diagnosis and monitoring. Exp Mol Pathol 86:186-191
7. Zapf I, Fekecs T, Ferencz A, Tizedes G, Pavlovics G, Kálmán E, Lõrinczy D (2011) DSC analysis of human plasma in breast cancer patients. Thermochim Acta 524:88-91

8. Todinova S, Krumova S, Gartcheva L, Robeerst C, Taneva SG (2011) Microcalorimetry of blood serum proteome: a modified interaction network in the multiple myeloma case. Anal Chem 83:7992-7998

9. Todinova S, Krumova S, Kurtev P, Dimitrov V, Djongov L, Dudunkov Z, Taneva SG (2012) Calorimetry-based profiling of blood plasma from colorectal cancer patients. Biochim Biophys Acta 1820:1879-1885

10. Tsvetkov PO, Tabouret E, Roman AY, Romain S, Bequet C, Ishimbaeva $\mathrm{O}$, Honoré S, Figarella-Branger D, Chinot O, Devred F (2018) Differential scanning calorimetry of plasma in glioblastoma: toward a new prognostic/monitoring tool. Oncotarget 9:9391-9399

11. Hennig C, Meila M, Murtagh F, Rocci R (2015) Handbook of cluster analysis. CRC Press, Boca Raton

12. Sarstedt M, Mooi E (2014) Cluster analysis. In: A concise guide to market research. Springer Texts in Business and Economics, Heidelberg

13. FindClusters-Wolfram Language Documentation. http://reference.wolfram.com/lan guage/ref/FindClusters.html?view=all

14. Mureşan M (2017) Introduction to Mathematica $^{(\mathbb{R}}$ with applications. Springer, Heidelberg 